\title{
Characterization of high molecular weight compounds in urban atmospheric particles
}

\author{
V. Samburova, R. Zenobi, and M. Kalberer \\ Department of Chemistry and Applied Biosciences, Swiss Federal Institute of Technology Zurich (ETH), 8093 Zurich, \\ Switzerland
}

Received: 1 November 2004 - Published in Atmos. Chem. Phys. Discuss.: 31 January 2005

Revised: 2 June 2005 - Accepted: 13 July 2005 - Published: 11 August 2005

\begin{abstract}
The chemical nature of a large mass fraction of ambient organic aerosol particles is not known. High molecular weight compounds (often named humic-like substances) have recently been detected by several authors and these compounds seem to account for a significant fraction of the total organic aerosol mass. Due to the unknown chemical structure of these compounds quantification as well as a determination of their molecular weight is difficult. In this paper we investigate water soluble humic-like substances in ambient urban aerosol using size exclusion chromatographyUV spectroscopy and laser desorption/ionization mass spectrometry (LDI-MS). LDI-MS was used for the first time to investigate HULIS from atmospheric aerosols. A careful evaluation of the two method shows that both methods complement each other and that both are needed to learn more about the molecular weight distribution and the concentration of humic-like substances. An upper molecular weight limit of humic-like substances of about $700 \mathrm{Da}$ and a concentration of $0.3-1.6 \mu \mathrm{g} / \mathrm{m}^{3}$ air can be estimated, corresponding to 9$30 \%$ of the total organic carbon for an urban background site.
\end{abstract}

\section{Introduction}

Atmospheric aerosol particles play an important role in many atmospheric processes, such as scattering and absorbing solar radiation or cloud formation (Ramanathan et al., 2001; Breon et al., 2002). Aerosol particles smaller than $10 \mu \mathrm{m}$ can penetrate deep into the human respiratory tract causing negative health effects (e.g., Dockery et al., 1993). Chemical components and physical properties of the aerosol particles critically influence these effects.

Atmospheric aerosols contain a significant fraction of organic components, up to 50\% in mass (Seinfeld and Pan-

Correspondence to: M. Kalberer

(kalberer@org.chem.ethz.ch) dis, 1998). Although hundreds of compounds were identified, the chemical nature of the vast majority at the organic mass remains unknown. It was postulated that high molecular compounds contribute significantly to the total organic aerosol mass (up to about 30\%) (Facchini et al., 1999). Such substances have chemical characteristics that are similar to humic acids (HA) and fulvic acids (FA) found in natural waters and soil, and the term "humic-like substances" (HULIS) was suggested (Havers et al., 1998). Several authors have analyzed this class of compounds, mainly using size exclusion chromatography methods (SEC) (Fuzzi and Zappoli, 1996; Zappoli et al., 1999; Krivácsy et al., 2000) or spectroscopic methods such as diffuse reflectance infrared Fourier transform spectroscopy (DRIFTS), UV or fluorescence spectroscopy (Havers et al., 1998; Zappoli et al., 1999; Kiss et al., 2003). SEC is also a widely used and well established technique in the field of humic and fulvic acid analysis (Rausa et al., 1991; Piccolo et al., 2001; Perminova et al., 2003; Janos, 2003).

Currently, only a few papers report an estimation of the molecular weight range and the concentration of high molecular weight compounds in atmospheric aerosols. Zappoli et al. (1999) estimated the concentration of HULIS by SEC with UV-VIS detection and found $0.6-2.5 \mu \mathrm{g} / \mathrm{m}^{3}$ HULIS in aerosol with an upper limit for the molecular weight of 3000 Da using humic acid as standard. Krivácsy et al. (2000) also used SEC with UV and fluorescence detection to investigate atmospheric HULIS, confirming the previous results of Zappoli et al. (1999) concerning the chemical similarity of HULIS and HA. The molecular weight distribution of HULIS was estimated by Kiss et al. (2003) with liquid chromatography - electrospray ionization mass spectrometry (HPLC-ESI-MS). These authors conclude that the molecular weight range of water-soluble macromolecular compounds is not higher than $500 \mathrm{Da}$.

(C) 2005 Author(s). This work is licensed under a Creative Commons License. 
In this study we critically evaluate calibration procedures and present results of molecular weight range determinations of water soluble HULIS extracted from atmospheric particles by two different methods, SEC and laser desorption/ionization mass spectrometry (LDI-MS). Differences and limitations of the two methods are discussed and concentration estimates of HULIS are given using Suwannee River Fulvic Acid Standard (SRFA) as a standard.

\section{Experimental}

Atmospheric aerosol particles were sampled in downtown Zurich, Switzerland, at an urban background site in August 2002. Particles smaller than $10 \mu \mathrm{m}$ in diameter (PM10) were collected on pre-baked quartz fiber filters $(150 \mathrm{~mm}$ diameter) with a high volume sampling system at a flow rate of 500 1/min (Model DA 80, Digitel, Hegnau-Volketswil, Switzerland). Three samples were collected continuously during 5 days, on 16-21 August, 21-26 August, and 28 August-2 September, respectively. Filters were then packaged into aluminum foils and stored in polyethylene bags at $-28^{\circ} \mathrm{C}$ until analysis.

High molecular weight compounds in the aerosol samples are compared with Suwannee River Fulvic Acid Standard (SRFA), which was obtained from the International Humic Substances Society (IHSS). SRFA was chosen as calibration compound for a quantitative HULIS determination because it is widely used and chemically well characterized (e.g., Noyes and Leenheer, 1989; Leland et al., 1999). In addition, the molecular size distribution of SRFA is $<1000 \mathrm{Da}$ as determined by various methods, among them LDI-MS and MALDI-MS (Novotny and Rice, 1995; Brown and Rice, 2000; Leenheer et al., 2001; Kujawinski et al., 2002). The same molecular size range is expected for HULIS (Kiss et al., 2003).

\subsection{Extraction}

Six $15 \mathrm{~mm}$ diameter pieces of each filter were extracted with solvents of different polarity. In the literature extraction of HULIS from atmospheric particles is mostly described with basic solvents such as aqueous sodium hydroxide (Havers et al., 1998). In this study, samples were extracted with milliQ water, aqueous $0.1 \mathrm{M} \mathrm{NaOH}$, tetrahydrofuran (THF) or toluene, thus covering a range of polar and apolar extraction media. Filters were first soaked in the solvent during $2 \mathrm{~h}$ and then sonicated for $2 \mathrm{~h}$. The supernatant was then filtered (PVDF Filter Media, $0.45 \mu \mathrm{m}$ pore size, Whatman) or centrifuged depending on the type of analysis performed. SRFA standards were sonicated and filtered in the same way as the filter samples. For both analysis methods performed, size exclusion chromatography and laser desorption/ionization mass spectrometry, the toluene and THF extracts showed no detectable signals indicating the very po- lar properties of atmospheric HULIS. Thus only water and $\mathrm{NaOH}$ extracts are considered in the following.

\subsection{Size exclusion chromatography (SEC)}

High pressure size exclusion chromatographic measurements were performed using a Waters Ultrahydrogel 120 column (300 $\mathrm{mm} \times 7.8 \mathrm{~mm}$, hydroxylated polymetacrylate stationary phase), and a SHIMADZU System Controller (SCL-10Avp), a Solvent Delivery Module (LC-10Atvp) and a Diode Array Detector (SPD-M10Avp). UV spectra between 190-800 nm were recorded.

MilliQ water and $\mathrm{NaOH}$ extracts from the filter samples and from the SRFA standards were filtered with a PVDF filter $(0.45 \mu \mathrm{m}$ pore size, Whatman $)$ prior to SEC analysis. An aqueous buffer solution $\left(0.3 \mathrm{M} \mathrm{NaCl}, 0.03 \mathrm{M} \mathrm{NH}_{4} \mathrm{Cl}, \mathrm{pH} 10\right.$ 11 adjusted with $\mathrm{NH}_{4} \mathrm{OH}$ ) at a flow rate of $1 \mathrm{ml} / \mathrm{min}$ was used as eluent (Krivácsy et al., 2000). Molecular weight determination by SEC may suffer from secondary effects making a true molecular size determination difficult. The eluent composition mentioned above helps to minimize such secondary effects, i.e., the high alkalinity reduces hydrophobic interactions between the stationary phase and the analyte, and the high salt concentration in the eluent suppresses electrostatic interactions between the stationary phase and the analyte. However, it cannot be completely excluded that some secondary interactions still interfere with the size separation.

\subsection{Laser Desorption/Ionization Mass Spectrometry (LDI- MS)}

LDI-MS was performed to determine the molecular weight distribution of the compounds present in the ambient aerosol and to compare the results with the SEC measurements. The experiments were performed with a time-of-flight (TOF) mass spectrometer (Axima-CFR, KRATOS Analytical Shimadzu, Manchester, UK, laser wavelength $337 \mathrm{~nm}$, pulse width $3 \mathrm{~ns}$, repetition rate $10 \mathrm{~Hz}$, linear mode, delayed extraction optimized for $\mathrm{m} / \mathrm{z}=1000$ ).

Filter samples were extracted as described above and centrifuged to separate the dissolved compounds from filter fibers and soot particles (the remaining solution was yellowish-brown colored). For a direct comparison with SEC, the extracts were also filtered with the same filters as used for SEC analysis. After filtration, the LDI-MS spectra were essentially the same compared to the centrifuged samples, however, with slightly lower signal intensities. Due to the high sodium content of the $\mathrm{NaOH}$ extracts, the LDI-MS were dominated by the $\mathrm{Na}^{+}$adducts, and a poor overall signal intensity was observed. Thus only milliQ water extracts were measured with LDI-MS and compared with SEC. The SRFA standard solution was prepared by dissolving SRFA in milliQ water, and optionally mixed with matrix. 
For the LDI measurement, $50 \mu \mathrm{l}$ of the extracts were directly added to the target stainless steel plate, the water was evaporated in a dessicator and the measurement was performed without further sample treatment. Optimization of the mass spectrum measurement procedure lead to the following set of parameters. To assure a representative sampling, 1000 mass spectra were usually summed up, scanning over an area of about $0.25 \mathrm{~mm}^{2}$. The laser power had to be kept in a narrow range $(20-25 \mu \mathrm{J} /$ pulse) in order to obtain sufficient signal but avoid fragmentation and plasma formation. Lower laser powers did not result in a shift to higher masses, but only to lower signal intensities, an indication that fragmentation from larger compounds is likely not a serious problem. In addition, several masses up to $\mathrm{m} / \mathrm{z} 430$ were checked for post source decay, i.e., metastable fragmentation of a compound in the time-of-flight drift tube. No significant post source fragmentation for all investigated peaks was observed. To further check the possibility whether fragmentation during the measurement would significantly cause an apparent shift to smaller masses, the addition of various matrices to the sample, i.e., nicotinic acid, 2-(4-hydroxyphenolazo)benzoic acid (HABA), 6-aza-2-thiothymine (ATT), 3-aminoquinone (3 QA), 1,8,9-trihydroxyantracene (Dithranol), dihydroxybenzoic acid (DHB) and graphite particles was studied. In Fig. 1a and $\mathrm{b}$ a LDI and a MALDI (using DHB as matrix) mass spectrum of the same HULIS sample is shown, respectively. No shift of the overall mass spectrum to larger masses could be observed after addition of matrix. Instead the complexity of the spectra increased due to matrix peaks and matrix-analyte adducts. Most of the additional peaks seen in the MALDI spectra are also seen in mass spectra from pure matrix and are marked with an asterisks in the MALDI spectrum (Fig. 1b). Some additional isolated peaks are seen in the MALDI-MS, likely matrix-analyte adducts. Similarly, SRFA was measured with (MALDI) and without matrix (LDI) addition. No significant difference between the two spectra was observed.

The fact that matrix addition did not resulted in significantly higher signal intensities nor in a shift of the spectra to higher masses might be due to compounds present in the complex aerosol sample, such as dihydroxybenzoic acids (Graham et al., 2002), that act as matrices by absorbing the desorption laser light and assuring efficient ionization. This hypothesis is supported by the structure of common matrices, which are often oxidized aromatic compounds. Similar compounds could also be present in aerosol particles as oxidation compounds of aromatic compounds.

These test experiments suggest that fragmentation is not significant in LDI-MS and does not lead to an underestimation of the molecular weight distribution of HULIS. We thus conclude that no significant gain in chemical information results from employing MALDI. In fact, MALDI data suffer from interferences, and LDI-MS gives more easily interpretable data for HULIS.
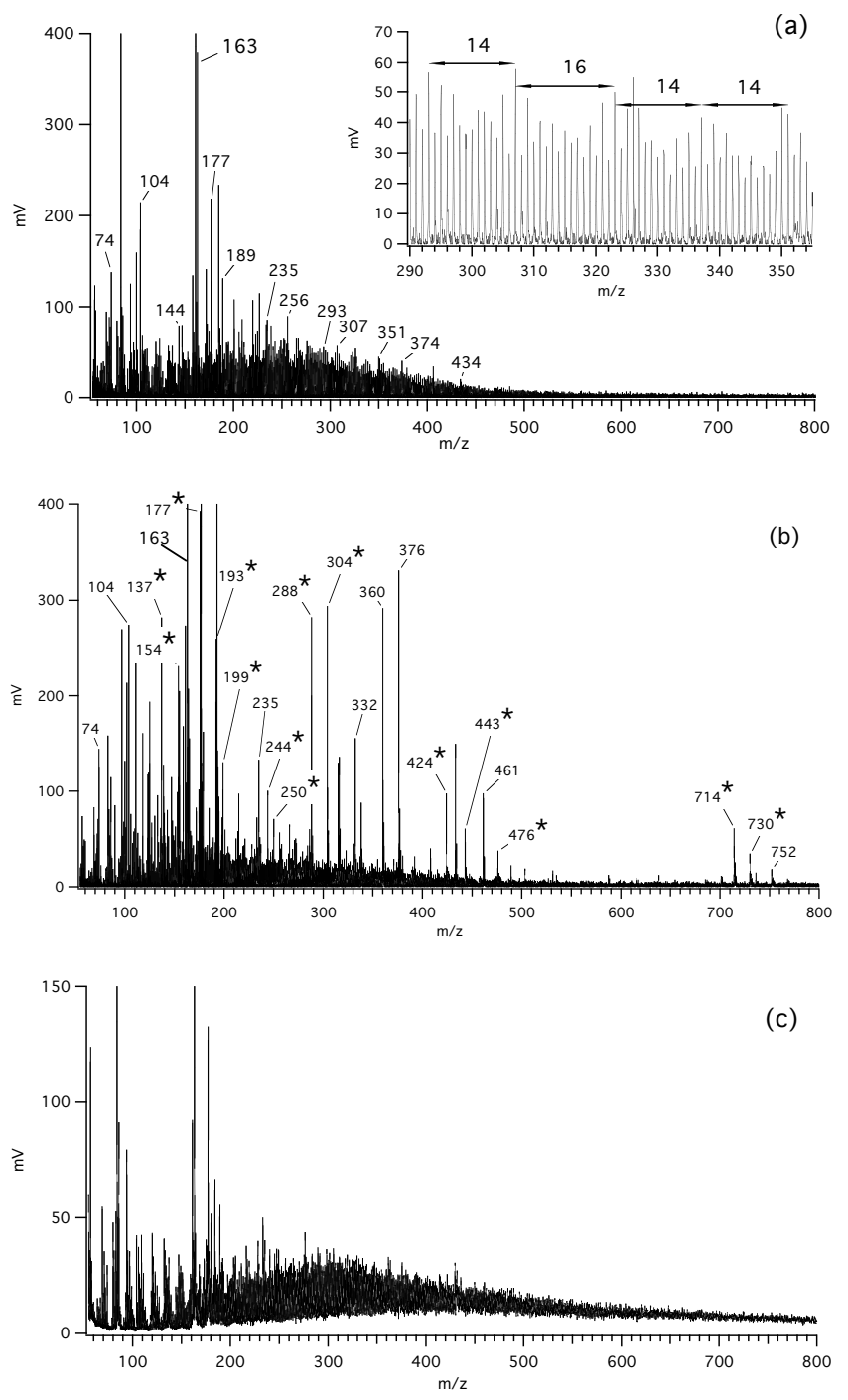

Fig. 1. LDI mass spectrum of (a) HULIS from a water extracted filter and (b) a MALDI mass spectrum using dihydroxybenzoic acid as matrix. The insert (a) shows an expanded view of the spectrum. Repetitive peak patterns with $\Delta \mathrm{m} 14$ and 16 are visible. Most of the additional peaks seen in the MALDI spectrum (b) are also seen in mass spectra from pure matrix and are marked with asterisks. (c) shows a LDI-mass spectrum of a filter sample, without prior extraction.

In addition, small filter pieces were measured with LDIMS without any further treatment in order to avoid artifacts due to extraction losses (Fig. 1c, see below for a discussion).

Besides fragmentation, multiply charged ions could potentially lead to measurement artifacts. However, multiply charged ions are typically observed in electrospray ionization while in laser desorption/ionization MS data are dominated by singly charged ions. Thus, it can be safely assumed that multiply charged ions are not abundant in the mass spectra presented here. 

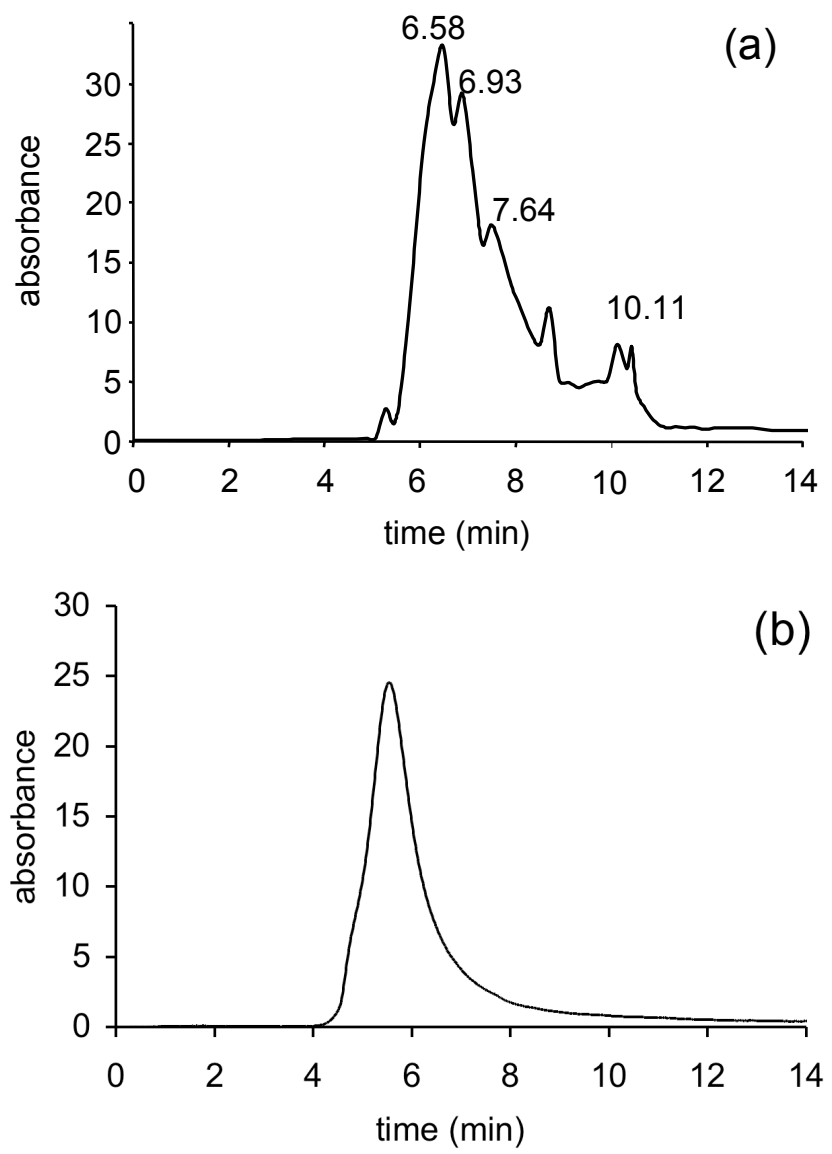

Fig. 2. Size-exclusion chromatograms of the $0.1 \mathrm{NaOH}$ extract of atmospheric aerosols collected in downtown Zurich (a) and fulvic acid standard (SRFA) (b) detected with UV absorption at $240 \mathrm{~nm}$.

\section{Results}

\subsection{SEC analysis}

There are a number of techniques that are used for measuring the molecular weight distribution of macromolecules such as size exclusion chromatography (SEC), field flow fractionation, ultrafiltration, ultracentrifugation or vapor pressure osmometry (Chin et al., 1991; Chin et al., 1994; Christl et al., 2000; Trubetskoj et al., 1997; Beckett et al., 1987; Perminova et al., 2003). We chose SEC not only because it is widely used but also because it is the most suited for measurements of high molecular weight compounds in atmospheric aerosols, because small amounts of sample can be analyzed, and because it is relatively fast. Also, SEC is well suited for separation and measurement of compounds with molecular weights below $5000 \mathrm{Da}$, the range expected for atmospheric HULIS, while other techniques are more appropriate for measurements of wider molecular weight ranges, and are therefore not easily applicable here. Considering the limitations mentioned in the experimental section, SEC is a

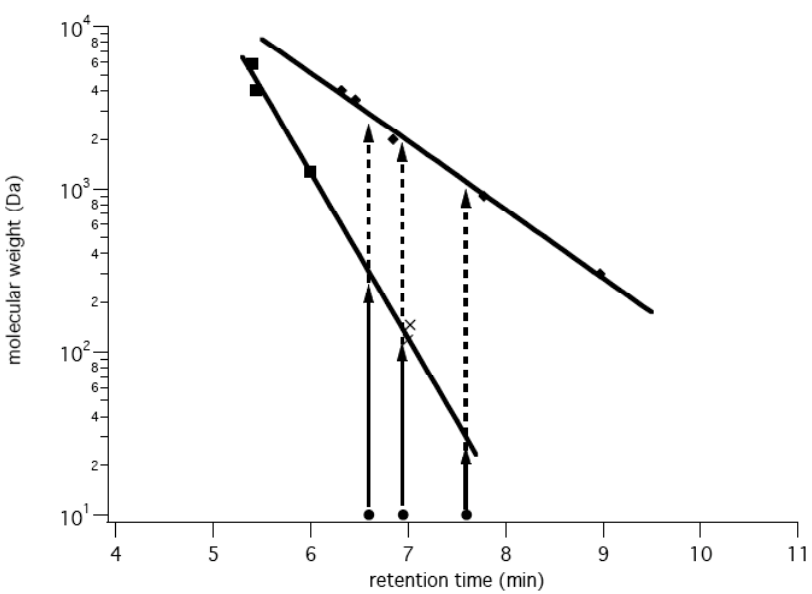

Fig. 3. Size calibration of SEC column with PEG ( $\checkmark)$, polymethacrylic acid $(\boldsymbol{\square})$, dicarboxylic acids $(\times)$ and retention times of HULIS $(\bullet)$

technique capable to separate organic compounds according to their molecular weight.

Size exclusion chromatograms of two extracts of the filter samples, in milliQ water and aqueous $0.1 \mathrm{M} \mathrm{NaOH}$, were compared. For both cases the size distribution of the extracted compounds is the same, but the peak intensity is about $30 \%$ higher for the $0.1 \mathrm{M} \mathrm{NaOH}$ extract, most likely due to the acidic character of HULIS making them more soluble in a basic extract than in water. The SEC analyses of all three filter samples show the same peaks with retention times between $5.5 \mathrm{~min}$ and $10.5 \mathrm{~min}$ recorded at $240 \mathrm{~nm}$ (Fig. 2a). The relative peak intensities are also the same for all filters with the exception of the peak at $10.1 \mathrm{~min}$ (see Fig. 2a), which in sample number 2 (21-26 August) was three times larger than for the two other samples. However, at these high retention times only small compounds are expected, which are not the focus of this paper. The main peaks in the high molecular weight range are observed at $6.58,6.93$, and $7.64 \mathrm{~min}$, respectively. The chromatogram of SRFA standard shows a monomodal distribution with the highest intensity at $5.56 \mathrm{~min}$ (Fig. 2b).

Several standards of different polarities were used for a molecular weight calibration, i.e., non-ionogenic polyethylene glycols (PEG), ionogenic polymethacrylic acids (PMA) and dicarboxylic acids (succinic, adipic and acelaic acid). A sufficient number of standards of these compounds were commercially available in the molecular size range below $4000 \mathrm{Da}$, needed for the HULIS calibration. Polystyrene sulfonates (PSS) that are often used as size calibration compound for fulvic acid (Perminova et al., 1998, 2003; Chin et al., 1994) was not applicable since not enough standards below $4000 \mathrm{Da}$ are available to generate a reliable calibration curve. For PEG and PMA standards with the same nominal mass, retention time differences of up to two minutes 
are measured as shown by the linear regressions in Fig. 3 . For an unknown compound this translates into large uncertainties for its molecular weight. For example, a retention time of $6.5 \mathrm{~min}$ corresponds to $500 \mathrm{Da}$ and $3500 \mathrm{Da}$ for the PMA and PEG calibrations, respectively. Such differences in size calibration depending on the chemical properties of the calibration compounds are well known e.g., Perminova et al. (1998), De Nobili and Chen (1999). The carboxylic acids fit well into the calibration curve of PMA due to their very similar chemical structure. This size calibration comparison shows drastically that determining the molecular weight of high molecular weight compounds with unknown chemical structure has to be performed with great care and that either theoretical considerations or results from other experimental techniques are necessary to provide additional information on the true molecular size distribution of HULIS.

The described discrepancies of the size calibration are due to the chemical properties of the standards and their interaction with the stationary phase, resulting in a significantly lower retention time of PMA compared to PEG with the same nominal mass, a phenomenon that has been described in the literature (e.g., Perminova et al., 1998). This is partly explained by the high $\mathrm{pH}(\mathrm{pH}=11)$ of the eluent, which leads to a deprotonation of PMA, but also of the stationary phase of the SEC column (hydroxylated polymethacrylic acid). Electrostatic repulsion causes PMA to interact less with the stationary phase than PEG, which is neutral and is not affected by the surface charges of the stationary phase, resulting in the faster elution of PMA.

The retention times of the main peaks of the chromatograms shown in Fig. 2a are indicated as cycles on the $\mathrm{x}$-axis in Fig. 3. Using these two calibration curves the retention times of 6.6, 6.9 and 7.6 min correspond to 3300, 2400 and $1300 \mathrm{Da}$, respectively for a PEG-equivalent weight and to 400,170 and $40 \mathrm{Da}$, respectively for a PMA-equivalent weight.

HULIS are rather polar compounds, as indicated by the extraction results with apolar solvents and consistent with literature data (Zappoli et al., 1999). Decesari et al. (2000) define HULIS as polyacidic compounds using ion chromatographic separation. Thus, it is expected that HULIS are separated on the SEC column similar to PMA, also a polyacid compound. However, as can be seen in Fig. 2a and 3 some smaller peaks are present in the chromatogram of the aerosol sample that elute at retention time of $7.5 \mathrm{~min}$ and later (i.e., theoretical PMA-equivalent mass of $\leq 50 \mathrm{Da}$ ), which cannot be explained by the PMA calibration. Thus, compounds eluting late in the chromatogram seem to have a significantly lower polarity than the PMA standard. To get further insight into the most likely size distribution for these compounds other techniques, such as LDI-MS, have to be compared with SEC.

\subsection{LDI-MS analysis}

LDI mass spectra were recorded for water extracts of the filter samples. No significant differences between the LDI spectra of all three filters were found. A mass spectrum of one of the three samples is shown in Fig. 1a. A broad range of peaks between $\mathrm{m} / \mathrm{z} 100-550$ is detected. Signals with lower intensities are present up to $\mathrm{m} / \mathrm{z} 700$ but no peaks were observed at higher mass. The use of matrix to aid desorption/ionization did not change the size distribution, as discussed above. These findings are in good agreement with HPLC-Elecrospray-MS analyses of HULIS by Kiss et al. (2003), who found an upper mass limit of about $500 \mathrm{Da}$ for HULIS in rural aerosol samples.

In the LDI spectrum shown in Fig. 1a (insert) a sequence of peaks with a mass difference of 14 and 16 is observed in the mass $\mathrm{m} / \mathrm{z}$ range of 250-400. Recent studies found similar mass patterns in aerosols formed in smog chamber experiments and explained them partially with oligomers formed from small carbonyls or dicarbonyls forming acetals (Tolocka et al., 2004; Kalberer et al., 2004). The similar repetitive mass pattern ( $\Delta \mathrm{m} 14$ and 16) of these laboratory experiments and the spectrum shown here from field samples suggests that this kind of oligomers might also be present in ambient aerosols.

Comparing the molecular weight determination from the LDI-MS measurements with the results of the two SEC calibrations and also considering the theoretical arguments discussed above, one can conclude that for the high end of the mass distribution the PMA calibration fits reasonably well to the LDI-MS results. This indicates an upper mass limit of organic compounds in urban aerosols of about $700 \mathrm{Da}$ (equal to a retention time of $6.25 \mathrm{~min}$ for the PMA calibration). However, at this stage one cannot exclude that compounds with $\mathrm{m} / \mathrm{z}>700$ are present in the ambient particles. They may have a very low ionization efficiency or fragment significantly in the LDI process, so that they may not be detected with the current method.

\subsection{UV measurements}

UV spectra between 190-370 $\mathrm{nm}$ of the aqueous aerosol extract are shown in Fig. 4. Traces a, b and c show spectra obtained at retention times of $6.58,6.93$ and $7.64 \mathrm{~min}$, respectively. The spectra at all three retention times are very similar, i.e., light absorbing properties do not seem to vary greatly for the different molecular weight fractions of the aerosol components. The SRFA standard (Fig. 4d) also shows a similar absorption spectrum. The aerosol components and the SRFA exhibit the same absorption maximum at $207 \mathrm{~nm}$, as well as the same fast decrease of the signal at higher wavelengths. However, between 210 and 240 the HULIS spectra show a more pronounced decease than the SRFA standard.

These findings support results reported in the literature (Zappoli et al., 1999) where in addition also fluorescence and 


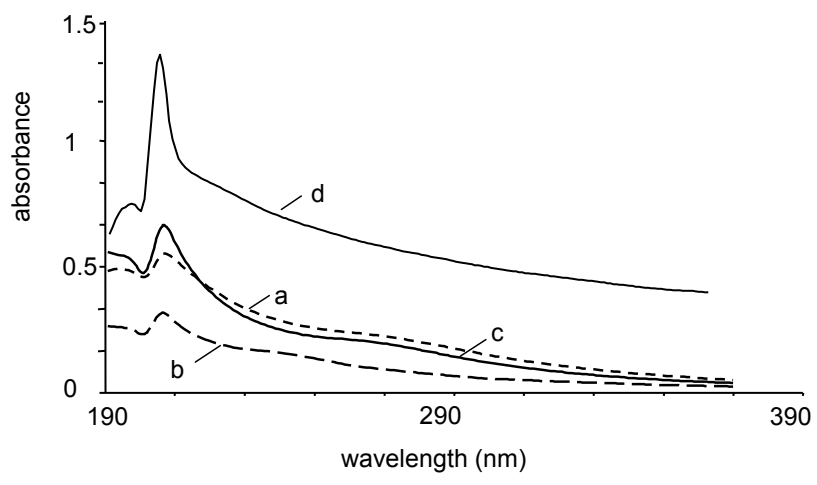

Fig. 4. UV spectra of HULIS at retention times of (a) $6.58 \mathrm{~min}$, (b) $6.93 \mathrm{~min}$ and (c) $7.64 \mathrm{~min}$ of the water extracted filter (see Fig. 2a) and of (d) a SRFA standard at 5.56 min retention time (see Fig. 2b).

diffuse reflectance infrared Fourier transform spectroscopy (DRIFTS) spectra of humic substances and HULIS were compared. All these measurements indicate that humic substances are suitable surrogates of HULIS in ambient aerosol.

\subsection{Concentration determination}

Based on the comparison described above a calibration curve with SRFA standards between $5.0-166.5 \mu \mathrm{g} / \mathrm{ml}$ was used to estimate the HULIS concentration in atmospheric aerosols. While statistical errors of the SEC measurement for quantification are small $(<2 \%)$, i.e., the major uncertainties originate from systematic errors. These need to be considered when using fulvic acid or specifically SRFA as surrogate standards to quantify HULIS, and are discussed in the following in detail.

(i) In ambient aerosol samples there are a number of inorganic water-soluble components that could interfere with the HULIS quantification. Sulfate and nitrate are by far the most abundant inorganic ions in ambient aerosols in Switzerland. In order to check this, sulfate and nitrate salts were measured with SEC. Nitrate has a retention time of $9 \mathrm{~min}$, which is outside the HULIS retention time window and thus does not interfere with HULIS. Sulfate eluting within the retention time window of HULIS is hardly absorbing UV at $240 \mathrm{~nm}$, the detection wavelength for the SEC. Sulfate concentrations equivalent up to $5 \mu \mathrm{g} / \mathrm{m}^{3}$, as measured for the same samples with ion chromatography, contributed less than $1 \%$ to the total UV absorbance in the HULIS retention time window. Thus, neither nitrate nor sulfate significantly influence the HULIS quantification with SEC.

(ii) Aerosol components identified so far on a molecular level (Seinfeld and Pandis 1998; Rogge et al., 1993) have molecular weights mostly smaller than about $300 \mathrm{Da}$ (e.g., alkanes up to $\mathrm{C} 21$ or 6-ring PAHs). Therefore we define all compounds with a molecular weight $>300 \mathrm{Da}$ as HULIS. Accordingly, only the respective retention time windows of 4.5-6.61 min and 4.5-8.93 min (corresponding to molecular sizes > $300 \mathrm{Da}$ for the PMA and PEG calibration), respectively, were used in the quantitative analysis.

(iii) Consequently also the respective mass fractions of SRFA within these two retention time windows were used for the calibration. This, however, requires that throughout the chromatogram the extinction coefficient of SRFA components stays relatively constant.

(iv) The extinction coefficient of the SRFA standard and HULIS (depending largely on the types and concentrations of chromophores) might be different for the same retention time window, which would introduce an error for the quantitative estimates. However, so far no better-defined alternatives to SRFA are available, and the quantitative comparison with the total organic carbon (see below) indicates that the current estimates are reasonable. Thus, the quantitative results obtained here can be considered as "fulvic acid equivalent" concentrations.

(v) Poor extraction efficiency of the HULIS from the filter material could lead to an underestimation of the HULIS concentration. Therefore extracted samples were compared with filter samples which were measured directly by LDIMS without extraction into a liquid. Figure 1c shows that the molecular weight distribution of such a direct LDI experiment is very similar to the extracted sample (Fig. 1a). Thus, it can be concluded that the extraction process is not substantially affecting the molecular weight distribution. However, spectra with poor mass resolution were obtained from these non-extracted samples, possibly due to electrical field distortion in the ion source caused by the rough filter surface.

Taking the above considerations into account the HULIS concentration (in SRFA-equivalents) in the three urban aerosol samples was $0.28-0.53 \mu \mathrm{g} / \mathrm{m}^{3}$ and $0.88-1.59 \mu \mathrm{g} / \mathrm{m}^{3}$ for the PMA and PEG calibration, respectively (Table 1). This amounts to $9-10 \%$ and $28-30 \%$ of total OC (Szidat et al., 2004), respectively in these samples. As discussed above, it seems more likely that the PMA-calibration is more representative for the HULIS quantification, thus HULIS concentrations seem to account for roughly $10 \%$ of the watersoluble OC in these samples. These HULIS concentrations are comparable to literature data, e.g. Zappoli et al. (1999) found HULIS concentrations of $0.6-2.5 \mu \mathrm{g} / \mathrm{m}^{3}$ for samples collected in Italy.

\section{Conclusions}

We investigated humic-like substances (HULIS) in atmospheric aerosols by two different techniques, i.e., size exclusion chromatography and laser desorption/ionization mass spectrometry. A comparison of LDI-MS and MALDI-MS using a large variety of matrices showed that matrix addition does not increase the observed molecular weight range of HULIS. Different compounds, i.e., polyethylene glycol (PEG), polymethacrylic acid (PMA), and dicarboxylic acids, were used as non-ionogenic and ionogenic standards for the 
Table 1. HULIS concentrations in atmospheric urban aerosol, quantified assuming different size calibration standards, and \% of HULIS of the total organic carbon.

\begin{tabular}{lllll}
\hline Sampling time & $\begin{array}{l}\text { SRFA-equivalent concentration } \\
\text { of HULIS }\left(\mu \mathrm{g} / \mathrm{m}^{3}\right) \\
(\text { PEG calibration) }\end{array}$ & $\begin{array}{l}\text { SRFA-equivalent concentration } \\
\text { of HULIS }\left(\mu \mathrm{g} / \mathrm{m}^{3}\right) \\
(\text { PMA calibration) }\end{array}$ & $\begin{array}{l}\% \text { of OC }\left(^{2}\right) \text { using } \\
\text { PEG calibration }\end{array}$ & $\begin{array}{l}\% \text { of OC }\left(^{2}\right) \text { using } \\
\text { PMA calibration }\end{array}$ \\
\hline 16-21 August 2002 & 1.59 & 0.53 & 28 & 9 \\
21-26 August 2002 & 0.88 & 0.28 & 30 & 10 \\
28 August-2 September 2002 & 1.49 & 0.47 & 30 & 9 \\
\hline
\end{tabular}

1 see text and Fig. 2

${ }^{2}$ OC data taken from Szidat et al. (2004)

molecular size calibration in the SEC experiments. Considering that HULIS are likely highly polar compounds (as extraction experiments with solvents of different polarity confirmed) the PMA and dicarboxylic acids are the better suited standards than PEG. Using the PMA calibration, an upper mass limit of about $700 \mathrm{Da}$ for HULIS in urban aerosols can be estimated. These results are in good agreement with our LDI-MS measurements, which showed a mass distribution in the same range. Direct LDI measurements of loaded filters, without extracting the aerosols showed a very similar molecular mass distribution, indicating that no substantial artifacts were introduced during the extraction work-up of the filter samples. Quantitative measurements, using Suwannee River fulvic acid as surrogate standard, showed that $0.3-1.6 \mu \mathrm{g} / \mathrm{m}^{3}$ of HULIS were present in the urban particles collected in summer 2002 , corresponding to about $10-30 \%$ of the total organic mater in the aerosol.

Acknowledgement. The support of the Swiss National Science Foundation (grant numbers 2169-061393.00/2 and 200020$103605 / 1)$ is greatly acknowledged. We thank C. Hüglin and M. Hill (EMPA) for the use of the high-volume sampler.

Edited by: Y. Rudich

\section{References}

Beckett, R., Jue, Z., and Giddings, J. C.: Determination of molecular weight distribution of fulvic and humic acids using flow fieldflow fractionation, Environ. Sci. Technol., 21, 289-295, 1987.

Breon, F. M., Tanre, D., and Generoso, S.: Aerosol effect on cloud droplet size monitored from satellite, Science, 295, 834-838, 2002.

Brown, T. L. and Rice, J. A.: The effect of laser wavelength and power density on the laser desorption mass spectrum of fulvic acid, Organic Geochem., 31, 627-634, 2000.

Chin, Yu-P. and Gschwend, P. M.: The abudance, distribution, and configuration of porewater organic colloids in recent sediments, Geochim. Cosmochim. Acta., 55, 1309-1317, 1991.

Chin, Yu-P., Aiken, G., and O'Loughlin, E.: Molecular weight, polydispersity, and spectroscopic properties of aquatic Humic Substances, Environ. Sci. Technol., 28, 1853-1858, 1994.
Christl, I., Knicker, H., Koegel-Knabner, I., and Kretzschmar, R.: Chemical geterogeneity of humic substances: characterization of size fractions obtained by hollow-fibre ultrafiltration, Eur. J. Soil Sci., 51, 617-625, 2000.

Decesari, S., Facchini, M. C., Fuzzi, S., and Tagliavini, E.: Characterization of water-soluble organic compounds in atmospheric aerosol: A new approach, J. Geophys. Res., 105, 1481-1489, 2000.

De Nobili, M. and Chen, Y.: Size exclusion chromatography of humic substances: limits, perspectives and prospectives, Soil Sci., 164, 825-833, 1999.

Dockery, D., Pope, C., Xu, X., Spengler, G., Ware, J., Fay, M., Ferris, B., and Speizer, F.: An association between air-pollution and mortality in 6 united-states cities, New Engl. J. Med., 329, 1753-1759, 1993.

Facchini, M. C., Fuzzi, S., Zappoli, S., Andracchio, A., Gelencser, A., Kiss, G., Krivacsy, Z., Meszaros, E., Hansson, H. C., Alsberg, T., and Zebuhr, Y.: Partitioning of the organic aerosol component between fog droplets and interstitial air, J. Geophys. Res., 104, 26821-26 832, 1999.

Fuzzi, S. and Zappoli, S.: The organic component of fog droplets, in: Proceedings of the 12-th International Conference on Cloud and Precipitation, Zurich, Switzerland, pp. 1077-1079, 1996.

Graham, B., Mayol-Bracero, O. L., Guyon, P., Roberts, G. C., Decesari, S., Facchini, M. C., Artaxo, P., Maenhaut, W., Koll, P., and Andreae, M. O.: Water-soluble compounds in biomass burning aerosols over Amazonia 1. Characterization by NMR and GC-MS, J. Geophys. Res., 107(D20), 8047, doi:10.1029/2001JD000336, 2002.

Havers, N., Burba, P., Lambert, J., and Klockow, D.: Spectroscopic characterization of humic-like substances in airborne particulate matter, J. Atmos. Chem., 29, 45-54, 1998.

Janos, P.: Separation methods in the chemistry of humic substabces, J. Chromat., 983, 1-18, 2003.

Kalberer, M., Paulsen, D., Sax, M., Steinbacher, M., Dommen, J., Prevot, A. S. H., Fisseha, R., Weingartner, E., Frankevich, V., Zenobi, R., and Baltensperger, U.: Identification of polymers as a major components of atmospheric organic aerosols, Science, 303, 1659-1662, 2004.

Kiss, Gy., Tombácz, E., Varga, B., Alsberg, T., and Persson, L.: Estimation of the average molecular weight of humic-like substances isolated from fine atmospheric aerosol, Atmos. Environ., 37, 3783-3794, 2003. 
Krivácsy, Z., Kiss, Gy., Varga, B., Galambos, I., Sárvári, Zs., Gelencsér, A., Molnár, Á., Fuzzi, S., Facchini M. C., Zappoli, S., Andraccio, A., Alsberg, T., Hansson, H. C., and Persson, L.: Study of humic-like substances in fog and interstitial aerosol by size-exclusion chromatography and capillary electrophoresis, Atmos. Environ., 34, 4273-4281, 2000.

Kujawinski, E. B., Hatcher, P. G., and Freitas, M. A.: Highresolution fourier transform ion cyclotron resonance mass spectrometry of humic and fulvic acids: improvements and comparisons, Anal. Chem., 74, 413-419, 2002.

Leenheer, J. A., Rostad, C. E., Gates, P. M., Furlong, E. T., and Ferrer, I.: Molecular resolution and fragmentation of fulvic acid by electrospray ionization/multistage tandem mass spectrometry, Anal. Chem., 73, 1461-1471, 2001.

Malcolm, R. L. and MacCarthy, P.: Limitations in the use of commercial Humic Acids in water and soil research, Environ. Sci. Technol., 20, 904-911, 1986.

Novotny, F. J., Rice, J. A., and Weil, D. A.: Characterization of fulvic acid by laser-desorption mass spectrometry, Environ. Sci. Technol., 29, 2464-2466, 1995.

Noyes, T. I. and Leenheer, J. A.: Proton nuclear magnetic resonance studies of fulvic acid from the Suwannee River: in Humic Substances in the SuwanneeRiver, Georgia: Interactions, Properties, and Proposed Structures, edited by: Averett, R. C., Leenheer, J. A., McKnight, D. M., and Thorn, K. A., U.S. Geological Survey Open-File Report 87-557, p. 231-250, 1989.

Perminova, I., Frimmel, F., Kovalevskii, D., Abbt-Braun, G., Kudryavtcev, A., and Hesse, S.: Development of a predictive model for calculation of molecular weight of humic substances, Water Res., 32 (3), 872-887, 1997.

Perminova, I., Frimmel, F., Kudryavtsev, A., Kulikova, N., AbbtBraun, G., Hesse. S., and Petrosyan, V.: Molecular weight characteristics of Humic Substances from different environments as determined by size exclusion chromatography and their statistical evaluation, Environ. Sci. Technol., 37, 2477-2485, 2003.
Piccolo, A., Conte, P., and Cozzolino, A.: Chromatographic and spectrophotometric properties of dissolved humic substances as compared to macromolecular polymers, Soil Sci., 166, 174-185, 2001.

Ramanathan, V., Crutzen, P. J., Kiehl, J. T., and Rosenfeld, D.: Atmosphere - Aerosols, climate, and the hydrological cycle, Science, 294 (5549), 2119-2124, 2001.

Rausa, R., Mazzolari, E., and Calemma, V.: Determination of molecular size distribution of humic acids by high-performance size-exclusion chromatography, J. Chromat., 541, 419-429, 1991.

Rogge, W., Mazurek, M., Hildemann, L., Cass, G., and Simoneit, B.: Quantification of urban organic aerosols at a molecular-levelidentification, abundance and seasonal-variation, Atmos. Environ., 27, 1309-1330, 1993.

Seinfeld, J. and Pandis, S.: Atmospheric Chemistry and Physics, New York, 1998.

Szidat, S., Jenk, T., Gäggeler, H., Synal, H., Fisseha, R., Baltensperger, U., Kalberer, M., Samburova, V., Wacker, L., Saurer, M., Schwikowski, M., and Hajdas, I.: Source apportionment of aerosols - what can be learn from 14C measurements in different carbonaceous particle fractions?, Radiocarbon, 46, 475-484, 2004.

Tolocka, M., Jang, M., Ginter, J., Cox, F., Kamens, R., and Johnston, M.: Formation of oligomers in secondary organic aerosol, Environ. Sci. Technol., 38, 1428-1434, 2004.

Trubetskoj, O. A, Trubetskaya, O. E., Afanas/eva, G. V., Reznikova, O. I., and Saiz-Jimenez, C.: Polyacrylamide gel electrophoresis of soil humic acid fractionated by size-exclusion chromatography and ultrafiltration, J. Chromat., 767, 285-292, 1997.

Zappoli, S., Andracchio, A., Fuzzi, S., Facchini, M. C., Gelencser, A., Kiss, G., Krivacsy, Z., Molnar, A., Meszaros, E., Hansson, H. C., Rosman, K., and Zebuhr, Y.: Inorganic, organic and macromolecular components of fine aerosol in different areas of Europe in relation to their water solubility, Atmos. Environ., 33, 27332743, 1999. 